\title{
Imaging of synchronous multiple primary lung adenocarcinoma with concomitant EGFR and KRAS mutations: a case report and review of the literature
}

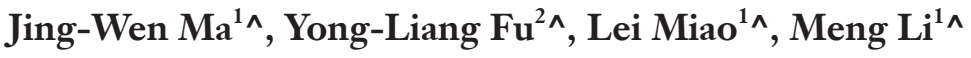 \\ ${ }^{1}$ Department of Diagnostic Radiology, National Cancer Center/National Clinical Research Center for Cancer/Cancer Hospital, Chinese Academy \\ of Medical Sciences and Peking Union Medical College, Beijing, China; ${ }^{2}$ Department of Pathology, National Cancer Center/National Clinical \\ Research Center for Cancer/Cancer Hospital, Chinese Academy of Medical Sciences and Peking Union Medical College, Beijing, China \\ Correspondence to: Meng Li, MD. Department of Diagnostic Radiology, National Cancer Center/National Clinical Research Center for Cancer/ \\ Cancer Hospital, Chinese Academy of Medical Sciences and Peking Union Medical College, No. 17 Panjiayuan Nanli Chaoyang District, Beijing \\ 100021, China. Email: lmcams@163.com.
}

\begin{abstract}
With the application of computed tomography (CT) imaging technology, the incidence rate of multiple primary lung cancer has gradually increased. However, the prevalence of concomitant epidermal growth factor receptor $(E G F R)$ and Kirsten rat sarcoma viral oncogene (KRAS) mutations in patients with non-small cell lung cancer (NSCLC) is low, and cases of concomitant EGFR and KRAS mutations have rarely been reported. In this case, we report a 71-year-old male patient with multiple primary lung adenocarcinoma harboring different gene mutation subtypes of KRAS and EGFR. The lesions in different lung lobes had distinct imaging features. One lesion was a solid mass in the upper lobe of the left lung, and the other was a cyst-like lung adenocarcinoma in the upper lobe of the right lung. Laboratory tests were positive for the marker carcinoembryonic antigen (CEA). The patient underwent thoracoscopic resection of the bilateral lung lesions, received chemotherapy and immunosuppressant therapy and exhibited progression-free survival (PFS) for 1 year. Later, the patient developed mediastinal lymph node and brain metastasis and died of multiple metastases. It is important to note that lung lesions with distinct imaging features may be associated with different types of gene mutations. Prediction of the gene mutation phenotype of lesions based on differences in imaging features and the biological behavior of genes, including the coexistence of EGFR and $K R A S$ mutations, will undoubtedly assist the clinical development of individualized diagnosis and treatment planning.
\end{abstract}

Keywords: Multiple primary lung cancer (MPLC); concomitant gene mutation; cystic lung cancer; case report

Submitted Nov 13, 2020. Accepted for publication Mar 24, 2021.

doi: $10.21037 /$ tcr-20-3258

View this article at: http://dx.doi.org/10.21037/tcr-20-3258

\section{Introduction}

Lung cancer continues to be the leading cause of cancerrelated death worldwide (1). As the most common histological form of lung cancer, lung adenocarcinoma is closely associated with oncogene mutations. Many studies show that computed tomography (CT) features are associated with genotypes $(2,3)$. However, research on the prediction of tumor mutation status from imaging finding, especially the gene phenotype of multiple primary lung cancer (MPLC) has not been performed. EGFR and KRAS are the two most common oncogenes

^ ORCID: Jing-Wen Ma, 0000-0002-0966-9179; Yong-Liang Fu, 0000-0002-4402-9732; Lei Miao, 0000-0002-7931-113X; Meng Li, 00000001-7592-2091. 
of lung adenocarcinoma and have received the most attention regarding the development of targeted therapy. Compared with other mutation types associated with lung adenocarcinoma, lung adenocarcinoma with EGFR mutation shows a good response to treatment with EGFR tyrosine kinase inhibitors (TKIs), such as gefitinib and erlotinib (4-6). However, KRAS mutation is still considered a poor prognosis factor. It is associated with resistance to TKIs and is considered an undruggable target, as efforts to therapeutically target $K R A S$ mutations have been unsuccessful (7). In clinical practice, EGFR mutations predict favorable progression-free survival (PFS), particularly in advanced non-small cell lung cancer (NSCLC) patients and these patients tend to have a better prognosis (7). In contrast, studies have shown that KRAS mutations are associated with a poor prognosis in patients with NSCLC, especially in patients with adenocarcinoma and early-stage NSCLC $(7,8)$. These two oncogenes were previously thought to be mutually exclusive in lung cancer patients (9).

In MPLC, prediction of the types of gene mutations by imaging features before treatment planning is crucial for individualized clinical treatment and can compensate for the lack of a pathological diagnosis. Here, we report a synchronous multiple primary lung adenocarcinoma with concomitant EGFR and KRAS mutations in which cystic lung adenocarcinoma was also found, and CT imaging characteristics are discussed as the key point.

We present the following article in accordance with the CARE reporting checklist (available at http://dx.doi. org/10.21037/tcr-20-3258).

\section{Case presentation}

The patient was a 71-year-old man with a history of tuberculosis whose father died of lung cancer. He had an approximately 52 -year history of smoking 20 cigarettes a day. Four years' prior, a left pulmonary nodule was incidentally found during a physical examination, and the patient visited our hospital for examination because of the recent development of a cough. The physical examination results showed no significant abnormalities except for a mild coughing symptom.

A CT scan showed two lung lesions, which indicated double primary lung cancer. Specifically, CT scans in June 2017 revealed bilateral lesions in the lungs, with a solid mass in the upper lobe of the left lung (Figure $1 A, B, C, D$ ) and cyst-like lung adenocarcinoma in the upper lobe of the right lung (Figure 1A,B,C,D), both of which were considered primary lung cancer. In detail, a superficial lobulated mass in the posterior segment of the tip of the left upper lobe, with a maximum cross-section of $2.4 \mathrm{~cm} \times 1.6 \mathrm{~cm}$, was visible and contained small empty bubbles, multiple spiculations on the edge and vacuoles at the local level. Moreover, there were several large vessels surrounding the lesion. In the right lung apex and posterior segment of the mediastinum, an irregular cystic lesion was visible with an unclear boundary and a local wide base attached to the mediastinal pleura. The maximum cross-section was approximately $4.2 \mathrm{~cm} \times 2.5 \mathrm{~cm}$. There were multiple ground-glass opacity (GGO) around the lesion. The wall of the local cystic cavity was thin with irregular margins, nodules on the wall were visible, and blood vessels were observed to be running through the lesion. Septations and ground-glass opacities were observed in the mass. No enlarged lymph nodes were found. The remaining lobes contained scattered small nodules.

Pathological results confirmed that both lesions were lung adenocarcinoma. The left lung mass consisted of poorly differentiated adenocarcinoma accompanied by neuroendocrine carcinoma differentiation (Figure 2A), and the right lung mass was moderately differentiated adenocarcinoma (Figure $2 \mathrm{~B}$ ). Immunohistochemical examination showed different types of gene mutations. The left lung tumor exhibited mutation of the KRAS gene at exon 2, codon 12 but no EGFR gene mutations in exons 18, 19, 20, or 21 (Figure 3A); the right lung mass contained a deletion mutation of the EGFR gene exon 19 but no mutation of the KRAS gene in exon 2, codon 12 or 13 (Figure $3 B$ ). Laboratory tests revealed an abnormal CEA tumor marker level, which returned to normal only at several days after the operation of left lung mass. It is worth mentioning that the patient's last blood CEA value in our hospital was increased. I don't know if it was a hematological indication of tumor recurrence. The CEA values were 10.07, 4.49, 4.25, 3.09, 10.05 and $22.73 \mathrm{ng} / \mathrm{mL}$ on June 26, 2017, August 17, 2017, September 16, 2017, November 7, 2017, March 26, 2018 and June 28, 2018, respectively (reference $0.0-5.0 \mathrm{ng} / \mathrm{mL}$ ).

The patient underwent thoracoscopic left upper lobectomy on July 4, 2017 and thoracoscopic right upper lobectomy with systematic lymph node dissection on September 19, 2017. The patient was in good condition at that time. The final review date of this patient was June 28, 2018 in our hospital. Then, the patient returned home for treatment in Harbin, the capital of Heilongiiang Province in 

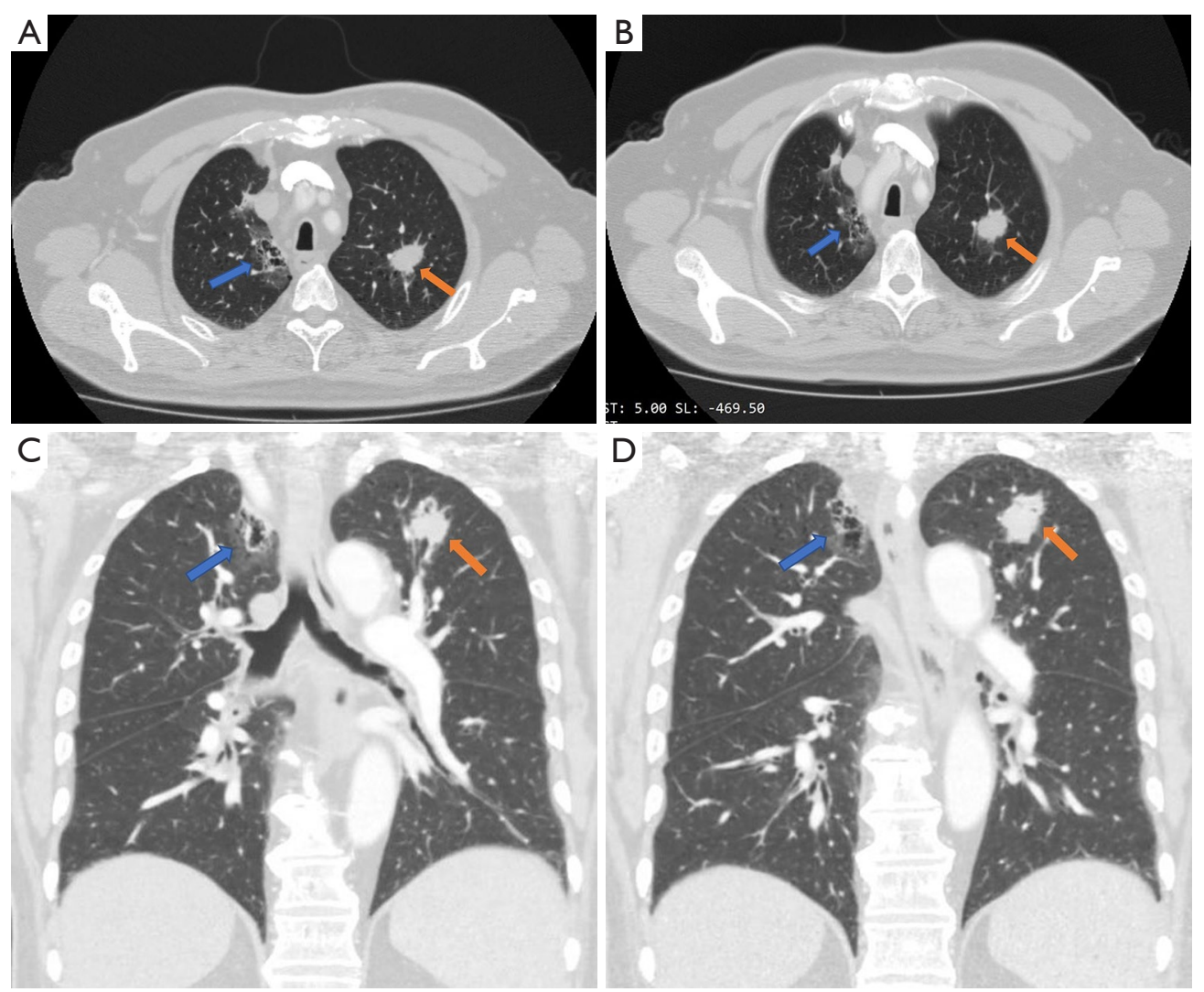

Figure 1 Computed tomography (CT) images. The blue arrow indicates the location of the right lung mass, and the orange arrow shows the left lung mass. The left solid lung cancer exhibits multiple burrs in the transverse axial view, and the right lung cancer has a cystic airspace in the transverse axial view at different levels (A,B). Lesions in both lungs are visible on different reconstructed coronal planes (C,D).
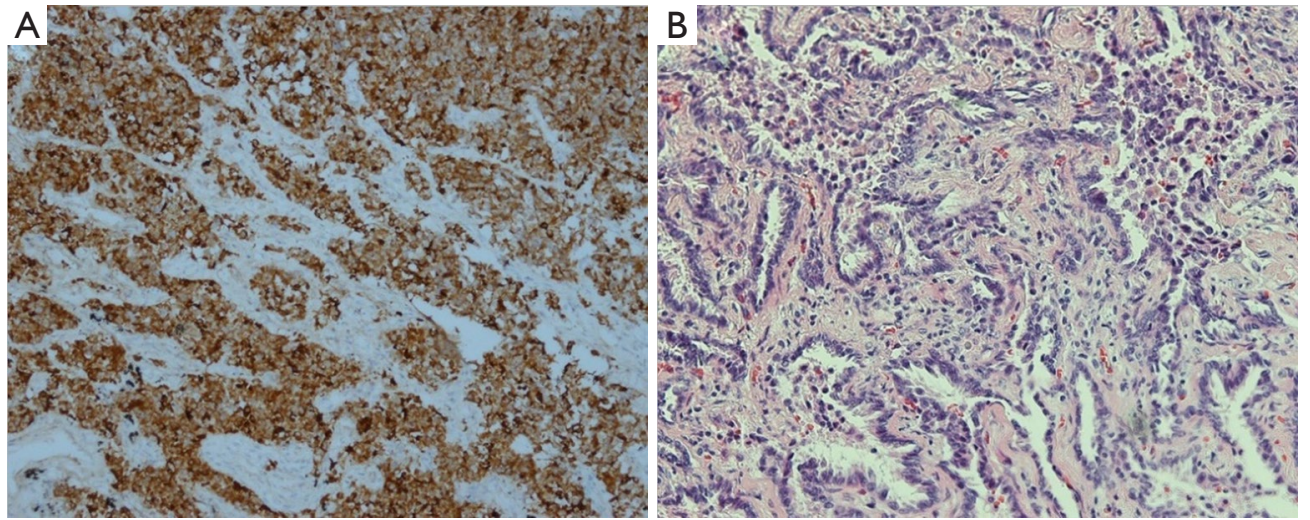

Figure 2 Pathological images. (A) Left pulmonary poorly differentiated adenocarcinoma with differentiation of neuroendocrine carcinoma, $\times 100$ with immunohistochemical staining; (B) right pulmonary moderately differentiated adenocarcinoma, $\times 400$ with hematoxylin-eosin staining. 


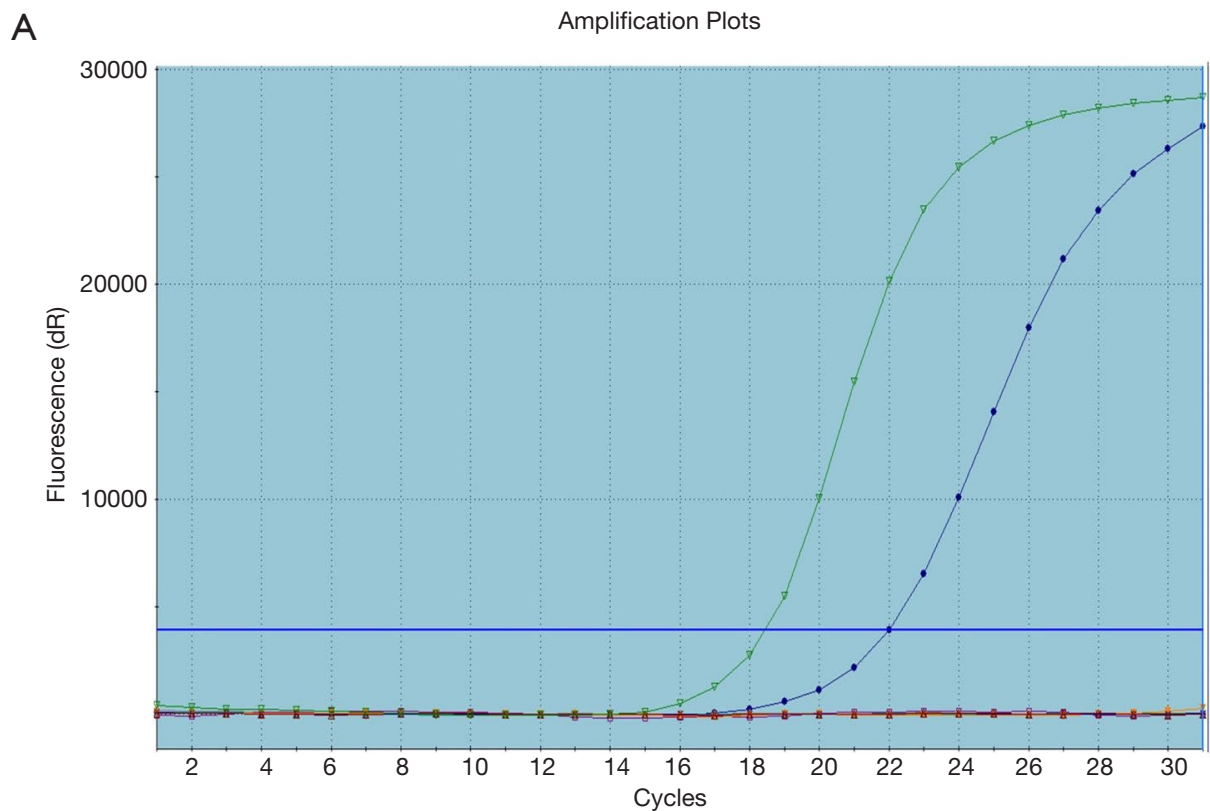

B

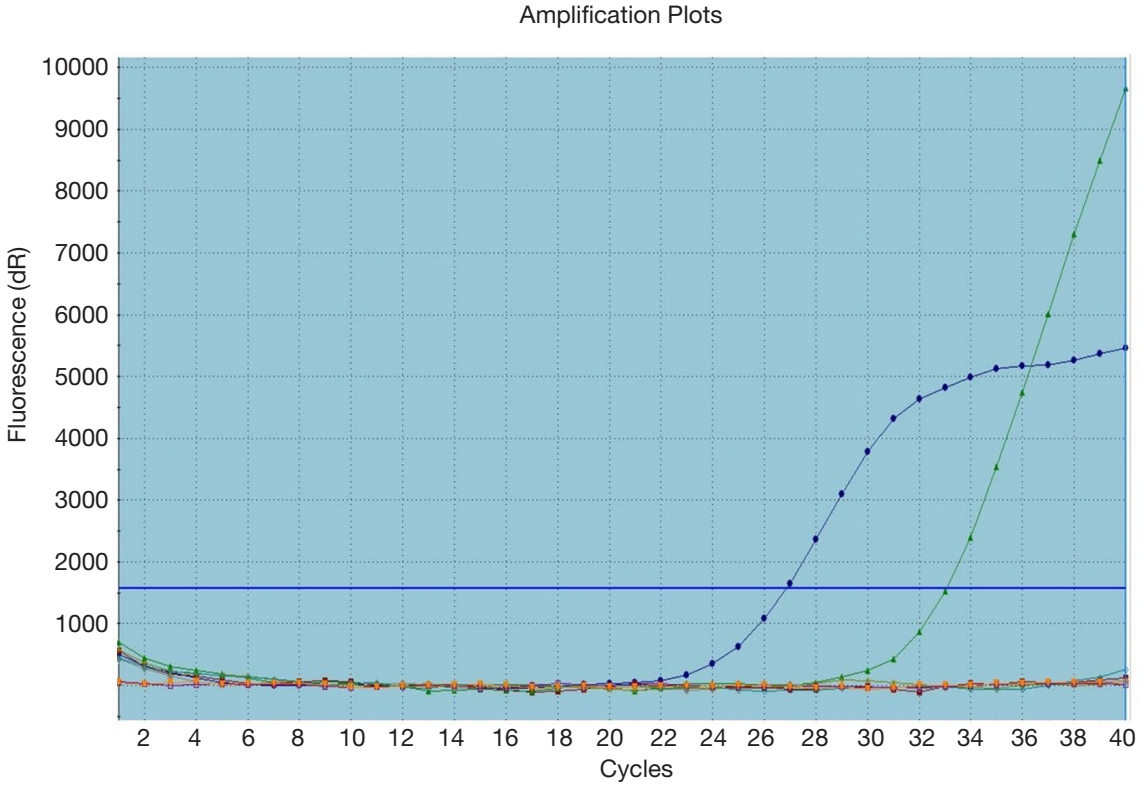

Figure 3 Detection of EGFR and KRAS mutations in multiple pulmonary nodules by real-time PCR. (A) KRAS mutation testing in the left lung lesion; (B) EGFR mutation testing in the right lung lesion.

China. According to the follow-up information, the patient developed mediastinal lymph node and left cerebellar mass metastasis according to an imaging diagnosis on September 6, 2018. Chemotherapy and immunosuppressant programmed cell death protein 1 (PD-1) therapy were performed for clinical treatment. The patient did not receive TKIs and died on October 28, 2019 because of multiple organ metastasis.

All procedures performed in studies involving human participants were in accordance with the ethical standards of the institutional and/or national research committee(s) and with the Helsinki Declaration (as revised in 2013). Written 
informed consent was obtained from the patient.

\section{Discussion}

To our knowledge, this is the first report to focus on CT imaging characteristics of MPLC displaying different EGFR and $K R A S$ molecular profiles with concurrent cystic lung cancer. At present, research on the pathogenic factors of lung cancer at the molecular level is being performed. In recent years, exploration of the imaging characteristics of different oncogenes has become a focus of research. The advantages of imaging, including that it is noninvasive, repeatable, economical and practical, can compensate for the lack of a pathological diagnosis, and most importantly, imaging will be beneficial for patients.

There are limited numbers of reports that have examined the association between EGFR/KRAS mutations and CT imaging features. However, current studies show differences in the imaging features correlated with EGFR and KRAS mutations. A meta-analysis and other original studies have demonstrated that a high GGO proportion is associated with $E G F R$ mutations in lung adenocarcinoma $(2,10-12)$. EGFR mutations are uncommon in the solid predominant subtype $(2,13)$. Smaller tumors (tumor diameter $\leq 3.0 \mathrm{~cm}$ ) with bubble-like lucency, homogeneous enhancement, or pleural retraction are independent predictive factors of EGFR mutations $(2,12)$. In contrast, KRAS mutations often occur in patients with large solid lesions (tumor diameter $>3.1 \mathrm{~cm})(14)$ that have no other distinct imaging features. The size features are inconsistent, but other imaging features of this case have been verified in cases of EGFR and KRAS mutations. In addition, convergence of the surrounding structure and multiple bilateral lung metastases are also CT features of EGFR-mutant tumors according to previous reports (15). Currently, clinical imaging data are insufficient to determine the mutation characteristics; therefore, further exploration of the potential molecular imaging features and large cohort studies or clinical trials should be performed to assist in clinical decision making. These markers would offer useful information for determining the appropriate treatment strategy of lung adenocarcinoma detected by CT when the patient cannot tolerate surgery or the specimen is not sufficient for genetic testing after pathological examination. Moreover, mutations in EGFR and KRAS show a mutually exclusive pattern (9), which also proves the case is bilateral MPLC with different origins. Comprehensive EGFR and KRAS gene mutation analysis can differentiate MPLC from metastasis and can better improve the currently used clinicopathological diagnostic criteria $(16,17)$.

MPLC is an important feature in this case. The incidence rate of MPLC has been increasing over the past few years with the increasing use of novel diagnostic methods, such as CT (18). MPLC represents diffuse pulmonary lesions with radiologic features on chest $\mathrm{CT}$ and genomic profiles that are important for preoperative diagnosis and clinical intervention planning. The problem of tissue sampling from such multiple small locations in the lung intrinsically remains, even with sophisticated CT-guided techniques. Moreover, identification of intrapulmonary metastases from MPLC still lacks uniform guidelines. Therefore, noninvasive and reproducible imaging features need to be explored, which could contribute to better prevention and earlier diagnosis. Lung cancer with intrapulmonary metastasis can be considered when the main lesion is a solid nodule with pleural attachment and the intrapulmonary accompanying lesion is also a solid nodule without lobules, speculation or bubble-like lucency (19). However, if the tumor is very small, it is still difficult to distinguish the primary cancer from intrapulmonary metastases according to the imaging characteristics. MPLC has been associated with better overall survival (OS) than lung cancer with intrapulmonary metastasis (20). For treatment, surgical resection remains the first choice for MPLC, and the maximum retention of lung function is also essential; thus, sublobectomy is now a commonly used surgical strategy. The $8^{\text {th }}$ WHO lung cancer staging system has previously proposed the standard diagnosis and staging mode of MPLC, which is very practical.

Another unique feature of this case is that the patient's right lung cancer was a cystic lung adenocarcinoma. It is likely that the widespread use of CT in daily clinical practice and the evolution of lung cancer screening programs have contributed to the increasing frequency of cystic lung adenocarcinoma encountered in imaging studies. Cystic airspaces in lung cancer are associated with smoking (21), and the patient had a 52-year history of smoking. Moreover, the most common pathologic type of cystic lung cancer is lung adenocarcinoma (22), as in this case. Cystic lung adenocarcinoma presents on CT as a "cystic area", containing air and a well-defined thin wall, which is the most common imaging feature; in addition, septation(s) can be observed within this area, and wall nodule(s), ground-glass opacities and irregular margins can be found around the area (22). All of the above radiologic features were observed in this case. Regarding clinical diagnosis, it is worth mentioning that the early signs of cystic lung adenocarcinoma are not 
obvious; therefore, it is easily misdiagnosed. The formation mechanism of cystic lung cancer is uncertain. However, to date, most studies tend to support the "check-valve" mechanism, which argues that the tumor originates from the alveolar wall and produces abundant fibrous tissue, resulting in narrowing and focal stenosis of the airway feeding that portion of the lung $(22,23)$.

There are several limitations of our report. First, we failed to observe the growth and imaging changes of the lung adenocarcinoma with $E G F R$ and $K R A S$ mutations because of rapid resection of the tumors. Second, the gene mutation type of the metastatic mediastinal lymph node was not determined. Provided that metastasis was driven by the EGFR mutation, TKIs could have prolonged the OS of the patient. Third, it is also a pity that we are unable to obtain the specific clinical treatment details of the patient after he returned home.

\section{Conclusions}

Overall, MPLC accompanied by EGFR and KRAS mutations, with the right lung cancer nodule presenting as cystic lung adenocarcinoma, is relatively rare. Early detection of tumor genotypes is challenging but very important for precise treatment and prognosis prediction. Additional cases are needed to precisely analyze the underlying mechanism that leads to different gene phenotypes exhibiting different imaging findings.

\section{Acknowledgments}

Funding: None.

\section{Footnote}

Reporting Checklist: The authors have completed the CARE reporting checklist. Available at http://dx.doi.org/10.21037/ tcr-20-3258

Conflicts of Interest: All authors have completed the ICMJE uniform disclosure form (available at http://dx.doi. org/10.21037/tcr-20-3258). The authors have no conflicts of interest to declare.

Ethical Statement: The authors are accountable for all aspects of the work in ensuring that questions related to the accuracy or integrity of any part of the work are appropriately investigated and resolved. All procedures performed in studies involving human participants were in accordance with the ethical standards of the institutional and/or national research committee(s) and with the Helsinki Declaration (as revised in 2013). Written informed consent was obtained from the patient.

Open Access Statement: This is an Open Access article distributed in accordance with the Creative Commons Attribution-NonCommercial-NoDerivs 4.0 International License (CC BY-NC-ND 4.0), which permits the noncommercial replication and distribution of the article with the strict proviso that no changes or edits are made and the original work is properly cited (including links to both the formal publication through the relevant DOI and the license). See: https://creativecommons.org/licenses/by-nc-nd/4.0/.

\section{References}

1. Siegel RL, Miller KD, Fuchs HE, et al. Cancer Statistics, 2021. CA Cancer J Clin 2021;71:7-33.

2. Liu Y, Kim J, Qu F, et al. CT Features Associated with Epidermal Growth Factor Receptor Mutation Status in Patients with Lung Adenocarcinoma. Radiology 2016;280:271-80.

3. Suh YJ, Lee HJ, Kim YJ, et al. Computed tomography characteristics of lung adenocarcinomas with epidermal growth factor receptor mutation: A propensity score matching study. Lung Cancer 2018;123:52-9.

4. Paez JG, Jänne PA, Lee JC, et al. EGFR mutations in lung cancer: correlation with clinical response to gefitinib therapy. Science 2004;304:1497-500.

5. Lynch TJ, Bell DW, Sordella R, et al. Activating mutations in the epidermal growth factor receptor underlying responsiveness of non-small-cell lung cancer to gefitinib. N Engl J Med 2004;350:2129-39.

6. Pao W, Miller V, Zakowski M, et al. EGF receptor gene mutations are common in lung cancers from "never smokers" and are associated with sensitivity of tumors to gefitinib and erlotinib. Proc Natl Acad Sci U S A 2004;101:13306-11.

7. Guan JL, Zhong WZ, An SJ, et al. KRAS mutation in patients with lung cancer: a predictor for poor prognosis but not for EGFR-TKIs or chemotherapy. Ann Surg Oncol 2013;20:1381-8.

8. Meng D, Yuan M, Li X, et al. Prognostic value of K-RAS mutations in patients with non-small cell lung cancer: a systematic review with meta-analysis. Lung Cancer 2013;81:1-10. 
9. Tam IY, Chung LP, Suen WS, et al. Distinct epidermal growth factor receptor and KRAS mutation patterns in non-small cell lung cancer patients with different tobacco exposure and clinicopathologic features. Clin Cancer Res 2006;12:1647-53.

10. Li M, Zhang L, Tang W, et al. Identification of epidermal growth factor receptor mutations in pulmonary adenocarcinoma using dual-energy spectral computed tomography. Eur Radiol 2019;29:2989-97.

11. Yang Y, Yang Y, Zhou X, et al. EGFR L858R mutation is associated with lung adenocarcinoma patients with dominant ground-glass opacity. Lung Cancer 2015;87:272-7.

12. Yano M, Sasaki H, Kobayashi Y, et al. Epidermal growth factor receptor gene mutation and computed tomographic findings in peripheral pulmonary adenocarcinoma. J Thorac Oncol 2006;1:413-6.

13. Song Z, Zhu H, Guo Z, et al. Correlation of EGFR mutation and predominant histologic subtype according to the new lung adenocarcinoma classification in Chinese patients. Med Oncol 2013;30:645.

14. Sugano M, Shimizu K, Nakano T, et al. Correlation between computed tomography findings and epidermal growth factor receptor and KRAS gene mutations in patients with pulmonary adenocarcinoma. Oncol Rep 2011;26:1205-11.

15. Hasegawa M, Sakai F, Ishikawa R, et al. CT Features of Epidermal Growth Factor Receptor-Mutated Adenocarcinoma of the Lung: Comparison with Nonmutated Adenocarcinoma. J Thorac Oncol 2016;11:819-26.

Cite this article as: $\mathrm{Ma} \mathrm{JW}, \mathrm{Fu} \mathrm{YL}, \mathrm{Miao} \mathrm{L}, \mathrm{Li}$ M. Imaging of synchronous multiple primary lung adenocarcinoma with concomitant EGFR and KRAS mutations: a case report and review of the literature. Transl Cancer Res 2021;10(5):2509-2515. doi: $10.21037 /$ tcr-20-3258
16. Girard N, Ostrovnaya I, Lau C, et al. Genomic and mutational profiling to assess clonal relationships between multiple non-small cell lung cancers. Clin Cancer Res 2009; 15:5184-90.

17. Girard N, Deshpande C, Azzoli CG, et al. Use of epidermal growth factor receptor/Kirsten rat sarcoma 2 viral oncogene homolog mutation testing to define clonal relationships among multiple lung adenocarcinomas: comparison with clinical guidelines. Chest 2010;137:46-52.

18. Romaszko AM, Doboszyńska A. Multiple primary lung cancer: A literature review. Adv Clin Exp Med 2018;27:725-30.

19. Zhou LN, Wu N, Zhao SJ, et al. HRCT features differentiate synchronous multiple primary lung adenocarcinomas from intrapulmonary metastases. Zhonghua Zhong Liu Za Zhi 2020;42:449-55.

20. Jiang L, He J, Shi X, et al. Prognosis of synchronous and metachronous multiple primary lung cancers: systematic review and meta-analysis. Lung Cancer 2015;87:303-10.

21. Fintelmann FJ, Brinkmann JK, Jeck WR, et al. Lung Cancers Associated With Cystic Airspaces: Natural History, Pathologic Correlation, and Mutational Analysis. J Thorac Imaging 2017;32:176-88.

22. Tan Y, Gao J, Wu C, et al. CT Characteristics and Pathologic Basis of Solitary Cystic Lung Cancer. Radiology 2019;291:495-501.

23. Xue X, Wang P, Xue Q, et al. Comparative study of solitary thin-walled cavity lung cancer with computed tomography and pathological findings. Lung Cancer 2012;78:45-50. 